\title{
Neurological and Developmental Outcome in Extremely Preterm Children Born in England in 1995 and 2006: The EPICure Studies
}

\author{
Tamanna Moore, Enid M. Hennessy, Jonathan Myles, Samantha J. Johnson, \\ Elizabeth S. Draper, Kate L. Costeloe, and Neil Marlow
}

\begin{abstract}
Academic Neonatology, UCL Institute for Women's Health (T.M., S.J.J., N.M.); and Wolfson Institute of Preventive Medicine, Queen Mary University of London, London (E.M.H., J.M.); Department of Health Sciences, University of Leicester, Leicester (E.S.D.); and Centre For Paediatrics, Blizard Institute, Barts and the London School of Medicine and Dentistry, Queen Mary University of London (K.L.C.); Homerton University Hospital NHS Foundation Trust, Homerton Row, London, UK (K.L.C.)
\end{abstract}

BMJ 2012;345:e7961

This is an open access article distributed under the Creative Commons Attribution License, which permits unrestricted use, distribution, and reproduction in any medium, provided the original work is properly cited.

\begin{abstract}
Survival rates for babies born extremely prematurely increased between 1995 and 2006, but few improvements in neonatal morbidity occurred despite appropriate interventions. The current study was undertaken to examine the neurologic and developmental outcomes for babies born at less than 27 weeks' gestation in 2006 and to compare the survival and outcomes at 3 years of age with those of babies born at 22 to 25 weeks' gestation in 1995.

Data were collected for all babies born at 22 to 26 weeks' gestation during 2006. Families were contacted for assessment when the children were aged 30 to 36 months. In 1995, data were collected for babies born at 22 to 25 weeks' gestation. Cerebral palsy was identified by neurologic examination and classified as severe, moderate, and mild, or no impairment in motor, developmental, sensory, and communication domains. Data from these 1995 and 2006 cohorts were combined to allow comparisons after reclassification of 2006 outcomes using the 1995 definitions.

A total of 576 children, aged 27 to 48 months, were evaluated in person. Information was available from local data records for another 191 children, aged 18 to 50 months, of whom 68 (38\%) had neurodevelopmental impairment. Of babies born at less than 27 weeks' gestation in 2006, 77 (13.4\%) had severe, and 68 (11.8\%) had moderate impairment. Rates for cognitive, communication, and motor impairment were $16 \%, 11 \%$, and $8 \%$, respectively. An inverse relationship was observed between gestational age and prevalence of moderate or severe impairment, that is, $45 \%$ of survivors at 22 to 23 weeks to $30 \%$ at 24 weeks, $25 \%$ at 25 weeks, and $20 \%$ at 26 weeks. Eighty-three children had cerebral palsy, 32 (39\%) with diplegia, $21(25 \%)$ with hemiplegia, $10(12 \%)$ with quadriplegia, and $20(24 \%)$ with other types. Nine children (11\%) with cerebral palsy had severe sensory impairment; developmental testing showed severe, moderate, or mild impairment in $47(57 \%), 30$ (46\%), and 6 (7\%) children, respectively. For births at less than 27 weeks' gestation in 2006, survival free of moderate or severe impairment ranged from $8 \%$ at 23 weeks' gestation to $59 \%$ at 26 weeks' gestation. Based on babies who received active intervention after birth, rates ranged from $11 \%$ at 23 weeks' gestation to $60 \%$ at 26 weeks' gestation and for babies receiving intensive care from $15 \%$ to $61 \%$, respectively.

When comparing to the historical cohort, survival to age 3 years for babies admitted to intensive care was $39 \%$ in 1995 and $52 \%$ in 2006. Overall, the proportion of babies admitted to intensive care who survived with severe disability increased by $2.6 \%$, but a higher proportion survived without disability (11\%). Survival without disability increased significantly at 25 and 24 weeks' gestation (15\% and $10 \%$, respectively), but changes were not statistically significant at 23 and 22 weeks' gestation $(2.5 \%$ and $-0.4 \%)$. In 1995, 43 children (18\%) had severe disabilities, and 54 (23\%) had other disabilities compared with $60(19 \%)$ and 54 (16\%), respectively, in 2006. Developmental scores of 55 or less were present in $92 \%$ in 1995 and 89\% in 2006. Mean scores for these children increased from 84 in 1995 to 91 in 2006.

The apparent improvement in survival and disability-free survival is encouraging but tempered by a lack of reduction in the prevalence of severe disability. The results show evidence of improvement in the proportion of babies who survive without disability, an improvement in developmental scores, and a reduction in neurologic morbidity, but no change in rates of severe impairment.
\end{abstract}




\section{EDITORIAL COMMENT}

(Historically, the advent of neonatal intensive care units (NICUs) has dramatically lowered the rate of neonatal mortality at low gestational ages. Around the world, in countries without the resources to have NICUs, babies born before 28 weeks' gestation have essentially a $100 \%$ rate of mortality. Although we have been able to push the threshold of viability down to 24 and even 23 or 22 weeks of gestation, the long-term morbidity of these neonates has continued to be quite severe and in a large proportion. There continue to be improvements made in NICU care with less aggressive use of high levels of oxygen or ventilation. Thus, the current article from the BMJ, abstracted above, examined the long-term outcomes at 3 years of age in neonates born at 22 to 25 weeks' gestation in 1995 and 2006.

This study found that survival rates between the 2 time periods were improved from $39 \%$ to $52 \%$ with a great dependence on the specific gestational age (ranging from $7 \%$ at 22 weeks up to $66 \%$ at 25 weeks). Discouragingly, there was no difference in the rate of severe morbidity in survivors. However, there was an increase in neonates who survived without disability from $23 \%$ to $34 \%$. Again, there was a strong relationship between gestational age and the prevalence of moderate or severe impairment (45\% of survivors at $22 / 23$ weeks to $25 \%$ at 25 weeks). However, much of this difference appeared due to improved survival rates as the rate of survival without severe disability was nonsignificantly increased from $59 \%$ to $65 \%$.

Although these data come from the United Kingdom, I think they are incredibly useful for counseling our patients here in the United States where long-term data are hard to come by. Given that about half of the survivors at 22 and 23 weeks' gestation have moderate or severe morbidity, which includes cerebral palsy, blindness, deafness, or cognitive delay, how should patients be counseled? Furthermore, how much autonomy over decisions regarding resuscitation should patients have at various gestational ages? In my conversations with colleagues around the country, nearly all would not even offer/allow resuscitation before 22 weeks. Alternatively, at 26 weeks and beyond, it is often not a parent choice because the neonatal outcomes are deemed to be so good. Between these gestations, I believe a range of practices exist from institutions where resuscitation before 24 weeks is not offered but done begrudgingly if parents inquire, to routine resuscitation of all 23 weeks' gestation neonates.

At my institution, patients undergo informed consent and choose resuscitation versus comfort care at 22 through 25 weeks' gestation. It is rare that we resuscitate a 22-week neonate and very rare that we don't at 25 weeks, but patients are given the opportunity to choose. Seemingly, this is better care than an absolute rule because it allows patients to incorporate their beliefs and preferences regarding this decision. Unfortunately, these patients often have only a short time to make the decision, and it is unclear how well they understand the severity of the long-term outcomes and even how to utilize the varying probabilities to make the decision. It is a very different decision than one about whether to undergo pregnancy termination when diagnosed with a Down syndrome fetus. In that case, patients know for certain that their fetus has Down syndrome, and although there is a range of outcomes, the range is relatively narrow compared with the outcomes at 22 to 25 weeks' gestation. Although the average outcome might be similar, the severe outcomes from 22- to 25-week neonates may be viewed as much worse than Down syndrome, and the survivors without disability have better outcomes than those with Down syndrome. So, not only do patients have to think about preferences toward the outcomes, but also they have to understand and use the probabilities related to the range of outcomes. I think this is an incredibly complex decision, one for which few expectant parents are prepared. Other than providing detailed information and responding to questions, we have little to help them make this monumental decision that can have enormous consequences. Furthermore, in this setting, it is not uncommon for these parents to interact with several different providers, most notably an obstetrician or MFM and a neonatologist. In my practice, I make every effort to do this counseling together to ensure a single message regarding this complicated information.-ABC) 